\title{
Best practice guidelines for the examination of specimens for the diagnosis of parasitic infections in routine diagnostic laboratories
}

\author{
J Francis, S P Barrett, P L Chiodini
}

J Clin Pathol 2003;56:888-891

Although most routine microbiology diagnostic laboratories process specimens for the diagnosis of parasitic infections, there are no best practice guidelines either for processing or for referral to specialist centres. Microscopy for parasites is most often requested on faecal samples, but urine, sputum, liver aspirates, duodenal aspirates, bile, corneal scrapings, contact lens fluid, and tissue are also encountered. Diagnosis of certain parasitic infections requires serology or polymerase chain reaction. These are undertaken in specialist laboratories, which should be consulted for expert advice on diagnosis and management of parasitic infections. Clinical Pathology Accreditation UK (CPA) has defined standards for assessing the quality of service provided by laboratories, but these do not include scientific and technical aspects. The Association of Medical Microbiologists has recently published Standards for laboratory practice in medical microbiology, which covers scientific and technical aspects, mainly bacteriological examination of specimens in routine diagnostic microbiology laboratories. These guidelines are complementary to the CPA standards and aim to ensure a consistent and high quality of service. This article provides best practice guidelines for the diagnosis of parasitic infections.

See end of article for authors' affiliations

Correspondence to: DrJ Francis, Department of Microbiology, Rotherham General Hospitals NHS Trust, Moorgate Road, Rotherham S60 2UD, UK; josephine.francis@ rothgen.nhs.uk

Accepted for publication 8 July 2003

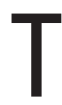
he examination for parasites by microscopy in routine diagnostic laboratories is mostly requested on faecal samples. Other specimens received for detection of parasites include urine, sputum, liver aspirates, duodenal aspirates, bile, corneal scrapings, contact lens fluid, and tissue. The use of serology for the diagnosis of parasitic infections is increasing. Occasionally, whole organisms or parts of organisms (for example, worms passed in stools or arthropods) are sent to the microbiology laboratory for identification.

Examination for plasmodia, microfilariae, and other parasites in the peripheral blood is usually carried out in the haematology laboratory. Tissues and biopsies for the diagnosis of parasitic diseases are processed in the histopathology laboratory. These two areas are not included in this article.

Certain parasitic infections (especially those that are extraintestinal) require serology or occasionally the polymerase chain reaction for their diagnosis. These are undertaken in parasitology specialist and reference laboratories/ units, and such units should be consulted for expert advice and assistance on the diagnosis and management of parasitic infections. This article provides general parasitology guidelines, followed by specific guidelines for the processing of non-faecal specimens for the diagnosis of parasitic infections.

\section{PROVISION OF DIAGNOSTIC PARASITOLOGY SERVICE \\ Standard}

There should be facilities for the detection of parasites on site. There should be trained staff available for the processing of parasitology specimens. An on call technical service should be provided. There should be a medical microbiologist available on call for clinical advice regarding the diagnosis and management of parasitic infections.

\section{Criteria}

- There should be provision of a parasitology service on site or suitable arrangements for such a service made with another unit.

- There should be arrangements in place for a specialised parasitology service with a parasitology specialist and reference laboratory.

- There should be out of hours access to clinical advice locally and/or for referral to specialised units for clinical advice on the diagnosis and treatment of malaria. (The identification of malarial parasites is not usually undertaken by microbiology laboratories.)

- Staff in general microbiology laboratories processing parasitology specimens should have appropriate initial training and should regularly update their knowledge by attending appropriate courses provided by parasitology specialist and reference units.

Abbreviations: HPA, Health Protection Agency; NEQAS, National External Quality Assessment Scheme 


\section{COLLECTION AND TRANSPORT OF SPECIMENS TO THE LABORATORY \\ Standard}

The specimens should be collected, transported, and stored, in a manner that will guarantee the best possible results within the testing systems available. ${ }^{1}$ Appendix A (tables $1-$ 3 ) provides a list of appropriate specimens and details of tests available in parasitology specialist and reference laboratories (User manual, Department of Clinical Parasitology and HPA Parasitology Reference Laboratory. Available on request from peter.chiodini@uclh.org).

\section{Criteria}

- Specimens collected for the examination of parasites should be appropriate for the clinical diagnosis of the infection-for example, fresh, unpreserved specimens of faeces for the examination of faecal parasites. Clear guidelines should be available to the users for the collection and transport of specimens to the laboratory.

- There should be local protocols for users regarding quantity, quality, and appropriateness of the specimens for the diagnosis of parasitic infections.

- Specimens from suspected or confirmed cases of viral hepatitis, tuberculosis, human immunodeficiency virus infection, and AIDS should be clearly identified as "danger of infection", according to local protocol.

\section{PROCESSING OF SPECIMENS}

\section{Standard}

Examination for parasites should be done by experienced staff, without undue delay. ${ }^{2}$

\section{Criteria}

- Examination of specimens for parasites should be performed by experienced staff. Such individuals should be processing a minimum number of parasitology specimens each year, personally processing any National External Quality Assurance Scheme (NEQAS) specimens, and should have undertaken specific parasitology training courses regularly and at least within the past five years.

- Specimens should be processed promptly or stored appropriately to prevent deterioration of morphology.

- Any unusual requests/specimens should be discussed with the consultant medical microbiologist.

- The method(s) used for the detection of parasites should be those that have achieved satisfactory NEQAS ratings.

- Parasites requiring further identification should be referred to a parasitology specialist and reference laboratory/unit.

\section{REPORTING OF RESULTS}

\section{Standard}

The consultant medical microbiologist or appropriate deputy should report all positive results promptly to the clinician. ${ }^{34}$

\section{Criteria}

- All important findings should be properly communicated to the clinician by the consultant medical microbiologist or appropriate deputy.

- Clinical advice on management of the infection and other investigations should be made available to the clinician.

- A final written report should follow all verbal/interim reports without delay.

\section{SEROLOGY OF PARASITIC INFECTIONS Standard}

There should be arrangements for the serological diagnosis of parasitic infection, with a parasitology specialist and reference laboratory/unit.

\section{Criteria}

- There should be a local protocol for the serological diagnosis of parasitic infection.

- There should be arrangements in place for the specialised serological diagnosis of parasitic infection with a parasitology specialist and reference laboratory/unit.

- Appropriate specimens of paired sera or single acute sera should be sent for such diagnosis.

- Specimens sent to specialist and reference laboratories should be packaged according to UN602 regulations.

\section{NOTIFICATION OF CLINICALLY RELEVANT RESULTS Standard}

There should be locally agreed protocols for reporting infections to the local Health Protection Agency (HPA) (public health teams).

\section{Criteria}

- Local protocols should be drawn up for the statutory reporting of relevant parasitic infections to the consultant in communicable disease control by the clinicians where appropriate.

- All results of epidemiological importance should be dealt with appropriately to prevent the spread of infection.

\section{CLINICAL ADVICE ON MANAGEMENT OF SUSPECTED/CONFIRMED CASES OF MALARIA Standard}

There should be 24 hour availability of an infectious disease physician or experienced consultant microbiologist (or some arrangement to access specialists) to give advice on the diagnosis and treatment of cases of malaria.

\section{Criteria}

- There should be 24 hour availability of a consultant medical microbiologist for clinical advice. There should be formal cover arrangements for absences (such as annual leave, etc) for such advice.

- There should be access to specialist clinical units for advice on the management of cases of malaria. Many infectious diseases units provide such a service-for example, the Hospital for Tropical Diseases provides clinical advice for the HPA malaria reference laboratory.

\section{EXAMINATION OF URINE FOR PARASITES} Standard

There must be provision for the examination of appropriate urine specimens for parasites.

\section{Criteria}

- A terminal urine specimen (the last $10-20 \mathrm{ml}$ passed) or alternatively a 24 hour collection of specimens of terminal urine is required for the diagnosis of Schistosoma haematobium infection. Egg excretion is highest around midday. Exercise before specimen collection is not considered necessary if the urine is passed around that time, but is helpful, along with fluid intake before micturition, in increasing egg excretion at other times of day.

- If there is a delay in the examination of urine, it is recommended that $0.5 \mathrm{ml}$ of $10 \%$ formalin is added to prevent the eggs of $S$ haematobium from hatching.

- A minimum of $10 \mathrm{ml}$ (10-20 ml) of terminal urine should be examined. 
- Additional specimens of urine, rectal snips, and/or rectal biopsy should be requested where appropriate.

- There should be standard operating procedures for the processing of urine specimens to achieve maximum recovery of parasites.

\section{EXAMINATION OF DUODENAL/JEJUNAL ASPIRATES AND BIOPSIES \\ Standard}

Appropriate specimens should be examined to detect parasites. Specimens should be processed according to standard procedures to achieve maximum recovery of parasites

\section{Criteria}

- There must be written protocols for the collection and transport of appropriate specimens to the laboratory.

- Specimens should be transported and processed without delay.

- If there is a delay in processing, the specimens should be refrigerated.

- The staining used must be validated.

\section{EXAMINATION OF LIVER ASPIRATES/LIVER BIOPSIES \\ Standard}

Adequate specimens of pus should be collected and transported to the laboratory promptly. Specimens should be examined without delay by experienced staff.

\section{Criteria}

- An aliquot of aspirated pus should be sent to the laboratory for examination for Entamoeba histolytica.

- Serology on peripheral blood is the method of choice to diagnose an amoebic liver abscess. Even in serologically confirmed cases, trophozoites of $E$ histolytica are very seldom seen in aspirated liver pus. However, if needle aspiration is carried out, the specimen should be transported and processed promptly to detect trophozoites of E histolytica.

- Specimens should be examined by experienced staff without delay. Liver biopsy specimens must be processed by the histopathology department.

\section{EXAMINATION OF CORNEAL TISSUE/CONTACT LENSES/CONTACT LENS FLUID FOR ACANTHAMOEBA SPP \\ Standard}

There should be arrangements in place for the provision of diagnosis of acanthamoeba infection in specimens from ophthalmology units.

\section{Criteria}

- There should be arrangements in place for the microscopic and culture diagnosis of Acanthamoeba spp infections.

- There should be arrangements in place for the serological diagnosis of Acanthamoeba spp infections. It is useful for the diagnosis of granulomatous amoebic encephalitis and other systemic acanthamoeba infections, rather than for the diagnosis of ocular disease.

\section{EXAMINATION OF BLOOD FILMS FOR MALARIA}

The examination of blood films for malaria is generally done by the haematology department, but the consultant medical microbiologist often provides clinical advice regarding the treatment of malaria. Any (haematology) laboratory that examines and reports blood films for malaria should consider having available an immunochromatographic strip test for non-microscopic diagnosis as a back up. Blood films found to contain malaria parasites should be sent to the HPA malaria reference laboratory for confirmation.

\section{Authors' affiliations}

J Francis, ULH NHS Trust, Grantham District Hospital, Grantham, Lincolnshire NG 31 8DG, UK

S P Barrett, Charing Cross Hospital, London W6 8RF, UK

P L Chiodini, The Hospital for Tropical Diseases, London WC1E 8BG, UK

\section{APPENDIX A}

Types of specimens and available tests for the diagnosis of parasitic infection. Some of these tests are available from parasitology reference laboratories/units.

\begin{tabular}{|c|c|}
\hline Parasites & \\
\hline \multicolumn{2}{|l|}{ Protozoa } \\
\hline Amoebae & Entamoeba histolytica and other amoebae \\
\hline Flagellates & Giardia lamblia (G intestinalis) \\
\hline Ciliates & Balantidium coli \\
\hline Coccidia & $\begin{array}{l}\text { Cryptosporidum parvum, Cyclospora cayetanensis, } \\
\text { Isospora belli, Sarcocystis spp }\end{array}$ \\
\hline Microspora & Microsporidia \\
\hline Helminths & Ascaris lumbricoides, Trichuris trichiura \\
\hline Nematodes & $\begin{array}{l}\text { Enterobius vermicularis, Ancylostoma duodenale, } \\
\text { Necator americanus, Strongyloides stercoralis }\end{array}$ \\
\hline Trematodes & $\begin{array}{l}\text { Schistosoma japonicum, S mansoni, S haematobium, } \\
\text { S mekongi, S intercalatum, Opisthorchis } \\
\text { (clonorchis), Fasciola hepatica, Fasciolopsis buski, } \\
\text { and other intestinal flukes, Paragonimus spp }\end{array}$ \\
\hline Cestodes & $\begin{array}{l}\text { Diphyllobothrium latum, Hymenolepis nana, Taenia } \\
\text { saginata/T solium }\end{array}$ \\
\hline
\end{tabular}

Table 2 Sample types other than faeces that can be tested and the parasites that can be detected

\begin{tabular}{ll}
\hline Specimen & Parasite \\
\hline $\begin{array}{l}\text { Adhesive tape slides } \\
\text { Duodenal aspirates }\end{array}$ & $\begin{array}{l}\text { Enterobius vermicularis } \\
\text { Giardia lamblia trophozoites, Strongyloides } \\
\text { larvae }\end{array}$ \\
Jejunal aspirates/ & $\begin{array}{l}\text { Microsporidial spores, Strongyloides stercoralis } \\
\text { larvae, Giardia lamblia trophozoites }\end{array}$ \\
Biopsies & Opisthorchis spp, Fasciola hepatica \\
Liver aspirates & Entamoeba histolytica \\
Cyst aspirates* & Echinococcus spp \\
Sputum & Strongyloides stercoralis/Paragonimus spp, \\
& Cryptosporidium spp \\
Cerebrospinal fluid & Acanthamoeba spp/Naegleria fowleri \\
Corneal scrapes & Acanthamoeba spp \\
Contact lens fluid & Acanthamoeba spp \\
Lymph node biopsies & Toxoplasma \\
Cutaneous ulcers & Leishmania spp \\
Bone marrow & Leishmania \\
Splenic aspirates & Leishmania \\
Liver biopsies & Leishmania \\
Muscle biopsy & Trichinella spiralis \\
Urine & Schistosoma haematobium \\
High vaginal swabs & Trichomonas vaginalis \\
Blood films & Plasmodium, microfilariae, babesia, \\
& trypanosoma \\
\hline
\end{tabular}

*The aspiration of cysts is hazardous and specialist advice should be sought regarding the management of hydatid cysts. 


\begin{tabular}{ll} 
Table 3 Serology: parasites and tests available \\
\hline Parasite & Tests for antibody detection \\
\hline Cysticercosis & Immunoblotting \\
Echinococcus & ELISA, CFT, immunoblotting \\
Entamoeba histolytica & LAT, CAP, IFAT \\
Fasciola & IFAT \\
Filaria & ELISA \\
Giardia & IFAT \\
Leishmania & DAT, rk 39 \\
Malaria & IFAT/ELISA \\
Schistosoma & ELISA \\
Strongyloides & ELISA \\
Toxocara & ELISA \\
Trichinella & IFAT \\
Trypanosoma (African) & IFAT \\
Trypanosoma (American) & IFAT, ELISA \\
\hline
\end{tabular}

CAP, cellulose acetate precipitins; CFT, complement fixation test; DAT, direct agglutination test; ELISA, enzyme linked immunosorbent assay; IFAT, immunofluorescence antibody test; LAT, latex agglutination test.

\section{APPENDIX B}

The following is a list of the Latin names (common names) of some frequently encountered parasites:

- Ancylostoma duodenale (hookworm)

- Ascaris lumbricoides (roundworm)

- Diphyllobothrium latum (fish tapeworm)

- Echinococcus granulosus (larval stage causes unilocular echinococcosis (cystic hydatid disease))

- Echinococcus multilocularis (larval stage causes multilocular echinococcosis (alveolar hydatid disease))

- Enterobius vermicularis (threadworm, pinworm)

- Fasciola hepatica (liver fluke)

- Fasciolopsis buski (intestinal fluke)

- Filarial worms (microfilaria cause filariasis)

- Wuchereria bancrofti (microfilariae cause bancroftian filariasis)

- Brugia malayi (microfilariae cause Malayan filariasis/ brugian filariasis)

- Brugia timori (microfilariae cause filariasis similar to that caused by $B$ malayi)

- Loa loa (microfilariae cause loiasis)

- Onchocerca volvulus (microfilariae cause onchocerciasis (river blindness))

- Hymenolepis diminuta (rat tapeworm)

- Hymenolepis nana (dwarf tapeworm)

- Leishmania spp (cause cutaneous, mucosal and visceral leishmaniasis (kala-azar))

- Necator americanus (hookworm)

- Clonorchis sinensis (Chinese liver fluke)

- Paragonimus westermani (lung fluke)
- Schistosoma spp (cause schistosomiasis (bilharzia))

$S$ haematobium (eggs cause primarily urinary symptoms) $S$ japonicum (eggs cause primarily intestinal symptoms) $S$ mansoni (eggs cause primarily intestinal symptoms) $S$ mekongi (eggs cause primarily intestinal symptoms) Sintercalatum (eggs cause primarily intestinal symptoms)

- Strongyloides stercoralis (larval form causes strongyloidiasis (intestinal and disseminated))

- Taenia saginata (beef tapeworm/adult worm causes intestinal infection-taeniasis)

- Taenia solium (pork tapeworm/larval stage causes cysticercosis; adult worm causes intestinal infection-taeniasis)

- Trichinella spiralis (larval stage causes trichinosis)

- Trichuris trichiura (whipworm)

- Trypanosoma brucei gambiense (causes West African trypanosomiasis-sleeping sickness)

- Trypanosoma brucei rhodesiense (causes East African trypanosomiasis-sleeping sickness)

- Trypanosoma cruzi (causes American trypanosomiasisChagas disease)

\section{REFERENCES}

1 Standard E3. CPA standards for accreditation Sheffield: Clinical Pathology Accreditation (UK) Ltd.

2 Standard F2. CPA standards for accreditation. Sheffield: Clinical Pathology Accreditation (UK) Ltd

3 Standard G1. CPA standards for accreditation. Sheffield: Clinical Pathology Accreditation (UK) Ltd.

4 Standard G3. CPA standards for accreditation. Sheffield, Clinical Pathology Accreditation (UK) Ltd (www.cpa-uk.co.uk).

\section{FURTHER READING}

1 Fleck SL, Moody AH. Diagnostic Techniques in Medical Parasitology. London: John Wright, 1988.

2 Chiodini PL, Moody AH, Manser DW. Atlas of medical helminthology and protozoology. 4th ed. Edinburgh: Churchill Livingstone, 2001.

3 Gutierrez Y. Diagnostic pathology of parasitic infections with clinical correlations. 2nd ed. New York: Oxford University Press, 2000

4 Ash LR, Orihel TC. Atlas of human parasitology. 4th ed. Chicago: American Society of Clinical Pathologists, 1997.

5 Public Health Laboratory Service. Standard operating procedure: investigation of specimens other than blood for parasites (B.SOP 31 Issue 1). London: PHLS, 1998.

6 Microbiology accreditation and quality assessment schemes in the UK: measuring up to the standards. Roberts C, Kelsey MC, eds. London: Association of Medical Microbiologists, 1992.

7 Medical and scientific staffing of National Health Service pathology departments. London: Royal College of Pathologists, June, 1999.

8 Preservation, retention and destruction of records, responsibilities of health authorities under the public records acts. London: Department of Health, Health Circular, $\mathrm{HC}(89) 20,1989$.

9 The retention and storage of pathology reports and archives. London: Royal College of Pathologists, Feb, 1999. 